\section{Epidemiology, risk factors and therapy of candidemia in pediatric hematological patients}

\author{
Chiara Cugno, Simone Cesaro \\ Pediatric Hematology Oncology, Azienda \\ Ospedaliera Universitaria Integrata \\ Verona, Italy
}

\section{Abstract}

Invasive fungal infections (IFI) are an important cause of morbidity, increased hospitalization and healthcare costs in critically ill or immunocompromised children. The mortality is comprised between 5 and $20 \%$. In the last 2 decades, the epidemiology of candidemia has changed with an increase of episodes caused by non-Candida albicans species. Central venous catheter, diagnosis of malignancy, and receipt of either vancomycin or antimicrobials with activity against anaerobic organisms for $>3$ days have been associated with the development of candidemia in the pediatric intensive care unit (PICU). Additional risk factors found in hematological patients were the diagnosis of aplastic anemia, performing an unrelated bone marrow or cord blood transplant, the occurrence of a graft versus host disease and the use of steroids. Early antifungal treatment is recommended to reduce mortality. In neutropenic patients, liposomal amphotericin B, an echinocandin (caspofungin, micafungin), and voriconazole are considered the best option especially for $C$. glabrata and $C$. krusei. Fluconazole remains a valid option for infection by Candida albicans in patients not exposed to fluconazole prophylaxis. Amphotericn B deoxycholate is generally not recommended because of its nephrotoxicity.

\section{Epidemiology}

Invasive fungal infections (IFI) are a considerable source of morbidity, mortality, increased hospitalization, and high healthcare costs in critically ill or immunocompromised children. ${ }^{1}$ Overall the incidence of sepsis due to fungal organisms increased by $207 \%$ in the last 20 years, ${ }^{2}$ with a similar trend in pediatric and adult patients. ${ }^{1}$ In children, Candida spp. represent the more frequent etiologic agent of sepsis by fungi. ${ }^{1}$ In a prospective study of pediatric nosocomial bloodstream infections (BSIs), Candida spp. were the third most common microbial cause of BSIs overall $(9.4 \%$ of isolates), and the most common fungal cause. ${ }^{3}$
The incidence of candidemia varies greatly between populations, with an incidence of 1.9 to 10.0 per 100,000 subjects reported in different population surveillance studies.4,5 Candidemia affects especially the neonatal than adults and older children and is frequently associated with signs and symptoms of sepsis syndrome.

The epidemiology of candidemia is changing with an increase in the proportion of episodes caused by non-Candida albicans species. This, in part, has been attributed to the broad use of azole as prophylaxis and therapy. ${ }^{6}$

The attributable mortality and morbidity associated with hospitalization remain significant (10.0\%-14.5\%) in all age groups despite therapeutic advances. ${ }^{1}$ Watson and coworkers reported that fungal sepsis was associated with the second highest case fatality (13.0\%) in children older than 1 year and up to 19 years old in 1995, just behind pneumococcal sepsis (14.5\%). ${ }^{7}$ The rate was higher in children aged 1 to 10 years $(16.8 \%)$ or 11 to 19 years $(11.6 \%)$ than in those younger than 1 year (10.8\%). Zaoutis and colleagues, in a large USA databases for adult $(n=8949)$ and pediatric ( $n=1118$ ) hospitalized patients, found that pediatric patients (median age 1 year, range 0 7) with a diagnosis of candidemia had a $10 \%$ higher rate of mortality ( $95 \% \mathrm{CI}=6.2-13.8)$ compared with matched candidemia-unexposed hospitalized patients. ${ }^{1}$ Data on the epidemiology and on the outcome of invasive fungal infections and candidemia in children with cancer are limited. Few studies have been conducted, often limited to patients with leukemia or HSCT. The incidence of IFI in this set of patients varies between $4,9 \%$ to $13,6 \%$ with mortality rate ranging from a minimum of $5 \%$ in patients with $\mathrm{ALL}^{8}$ to $59 \% .{ }^{9}$ In a prospective multicenter study in pediatric patients with cancer 0 after HSCT, Castagnola et al. reported an overall mortality of $28 \%$ for all causes of IFI and of $5 \%$ for fungemia (mostly of them were candidemia). ${ }^{10}$ In the most recent and largest series, Mor et al. found an IFI and candidemia incidence respectively of $7,2 \%$ and $1,4 \%$ in a population of 1047 children with hematological and oncologic disease or HSCT, and a mortality rate of $21,7 \% .11$

\section{Risk factors and prognosis}

Risk factors for fungal infection are similar in pediatric and adult patients. They include prolonged stay in an ICU, prior bacterial infection, use of a central venous line (CVC), total parenteral nutrition (hyperalimentation), immunosuppression (related to malignancy and its treatment, transplantation immunosuppressive therapy or other factors). Additional factors associated with elevated risk of invasive candidiasis are the use of mechan-
Correspondence: Simone Cesaro, Pediatric Hematology Oncology, Azienda Ospedaliera Universitaria Integrata, piazzale L.A. Scuro 10 , 37134 Verona, Italy.

Tel. +39.045 .8126889 - Fax: +39.045 .8124909 .

E-mail: simone.cesaro@ospedaleuniverona.it

Key words: candidemia, Candida spp. Sepsis.

Received for publication: 14 October 2011.

Revision received: 9 January 2012.

Accepted for publication: 10 January 2012.

This work is licensed under a Creative Commons Attribution NonCommercial 3.0 License (CC BYNC 3.0).

(C) Copyright C. Cugno and S. Cesaro., 2012 Licensee PAGEPress srl, Italy

Pediatric Reports 2012; 4:e9

doi:10.4081/pr.2012.e9

ical ventilation (endotracheal intubation), dialysis, extended vancomycin use, and recent surgery. 12 Children suffering from acute myeloid leukemia or relapsed leukemia or submitted to HSCT are at high risk for IFI. For pediatric patients receiving HSCT additional risk factors are represented by aplastic anemia, unrelated bone marrow or cord blood transplantation, graft versus host disease and steroid use. ${ }^{13}$ Zaoutis et al. found that the presence of a CVC, a diagnosis of malignancy, and receipt of either vancomycin or antimicrobials with activity against anaerobic organisms for $>3$ days were independently associated with the development of candidemia in the PICU. Children in the PICU with $\geq 3$ of these risk factors in different combinations had between $10 \%$ and $46 \%$ predicted probability of developing candidemia. ${ }^{14}$ In particular, the presence of a vascular access device or CVC seems to be one of the most important risk factor: candidemia may be associated to a vascular access device even in $70 \%$ of infected children. ${ }^{15}$ However, the source of Candida in a particular subgroup of patients suffering from neoplastic disease, more likely derives from the gastrointestinal tract due to mucosal disruption caused by cytotoxic chemotherapy and abrogation of normal gastrointestinal flora by antimicrobial therapy, which create a permissive environment that allows Candida to invade the mesenteric circulation. Candida infection can disseminate to end organs, including brain, lungs, liver, heart, kidneys, eyes, and spleen. Disseminated disease is associated with higher candidemia-related mortality. Risk factors for dissemination in a children with persistent candidemia are represented by CVC in place and immunosuppression.

In a recent study on epidemiology and outcome of candidemia in USA, it resulted that $C$. 
Albicans was detected in $45,6 \%$ of cases and that among $C$. non albicans $(54,4 \%$ of cases) the majority of species indentified included $C$. glabrata (26\%), C. parapsilosis (15,7\%), C. tropicalis $(8,1 \%)$ and $C$. krusei $(2,5 \%) .16$

Patients with $C$. glabrata and $C$. krusei candidemia were the most likely to have received prior antifungal therapy. This may reflect selective pressure caused by the extensive use of prophylactic fluconazole in susceptible hosts. Patients with $C$. krusei candidemia were younger and did not generally have such additional risk factors as parenteral nutrition, mechanical ventilation and concomitant bacterial infections. ${ }^{16} C$. parapsilosis is a common pathogen in neonates and children. 15 The incidence of $C$. parapsilosis infections has been attributed, in part, to the association among $C$. parapsilosis, catheter infection, and parenteral nutrition, because of its strong affinity for foreign material and its growth advantage in total parenteral nutrition solution. Transmission of C. parapsilosis from the hands of health care workers to neonates and children has been suggested as a contributor, because the organism is commonly carried on the hands of health care workers.

In both adult and pediatric patients, candidemia due to $C$. parapsilosis is associated with lower mortality rates than cases involving C. albicans, whereas crude mortality rates seem to be generally lower in pediatric than adult patients with candidemia regardless of the Candida spp. involved. 17

\section{Diagnosis}

Blood culture is the current gold standard for diagnosis of candidemia, although it is slow and lacks of sensitivity. Other tests, based on non-culture-based methods, such as mannan antigen and anti-mannan antibodies, may be useful for diagnosing invasive candidiasis. ${ }^{18}$ Patients with persistent positivity of blood cultures by Candida should be clinically assessed to exclude end-organ Candida diseases by ultrasound of liver, spleen, and heart, fundoscopy (eye) and if needed, by computed tomography or magnetic resonance.

\section{Therapy}

The outcome of candidemia largely depends on the early initiation of effective antifungal therapy. ${ }^{19}$ Inadequate initial therapy is associated with a significant increase in mortality. Delaying the initiation of antifungal therapy for more than 12 hours after withdrawal of the blood sample that yields Candida spp. may double the mortality compared to an earlier start of therapy. The decisional process for the treatment usually takes place in two steps: the first treatment is based on the positivity of blood culture for Candida spp. before species identification and may be subsequently modified according to identification of species and to their susceptibility profile.

Fluconazole, Amphotericin B deoxycholate, caspofungin and voriconazole are primary treatment options in non-neutropenic patients, as demonstrated in many randomized studies. ${ }^{20-22}$ On the other hand, only few data are available on neutropenic patients.

Since its introduction in the early 1990 s, fluconazole has been established as the most commonly used drug for invasive Candida infections. Amphotericin B preparations, caspofungin and voriconazole are primarily used for the treatment of patients with nonalbicans Candida infection andor patients with an increased risk for mould infections, such as haematological patients receiving immunosuppressive therapy or transplant recipients. The echinocandins represent the latest class of systemically active antifungals. They inhibit the synthesis of 1,3-beta-D-glucan, a key component of the fungal cell wall. Caspofungin was the first echinocandin to be approved by the FDA and the EMEA in 2001, followed by anidulafungin (2007) and micafungin (2008). As judged by randomized studies and pharmacological profiles, these drugs are valuable therapeutic options for invasive candidiasis. Caspofungin and micafungin are the 2 echinocandins approved for pediatric patients with invasive candidiasis.23,24 They have been studied in children with invasive candidiasis. Published caspofungin data are mostly limited to neonatal candidiasis, ${ }^{25}$ although its use in management of invasive candidiasis was retrospectively described within larger pediatric cohort studies. ${ }^{26,27}$

Efficacy and safety of the echinocandins were investigated in randomised, double blinded clinical trials in patients with candidemia and other forms of invasive Candida infections. Rex et al in a randomized study performed in early 90 ', established the equivalent efficacy of amphotericin B and fluconazole. ${ }^{21}$ On these basis, micafungin and caspofungin has been tested in comparative randomised trial with liposomal amphotericin B, although liposomal amphotericin B is not approved as first-line treatment of invasive Candida infections. These studies established the non-inferiority of micafungin and caspofungin to standard regimens.22,28-30 Caspofungin was as effective as amphotericin B, and the efficacy of micafungin was comparable with liposomal amphotericin B and caspofungin. In addition significantly less adverse effects occurred in the echinocandin arms compared with amphotericin B formulations. Micafungin also demonstrated to be safe and effective in ran- domized, double-blind, comparison trial in children with invasive candidiasis and in an open-label, noncomparative trial for newly diagnosed or refractory candidiasis. ${ }^{31,32}$ The randomised trial of anidulafungin $v s$ fluconazole was the first to demonstrate the superiority of an antifungal drug $v s$ a standard therapeutic regimen in invasive Candida infections, with, in addition, a safer profile in terms of hepatic toxicity. ${ }^{32}$ Echinocandins rarely interact with other compounds and their application is not affected by renal function, while hepatic insufficiency requires moderate dose adjustment only with caspofungin. Therefore echinocandins may represent the treatment of choice for intensive care patients, who usually suffer from multiple organ failure and receive broad concomitant medication with complex drug-drug interactions. Among the echinocandins, anidulafungin, which is not licensed for the pediatric use, appears to have the least propensity of drug interactions and does not require any dose adjustments. It thus may confer an additional benefit in patients receiving immunosuppressants or inducers of hepatic metabolism and those with liver insufficiency.

In Table 1 the pediatric dosages and the grade of recommendation for neutropenic patients is showed. The 2009 guidelines for candidiasis management from the Infectious Diseases Society of America (IDSA) recommended the empiric treatment for critically ill patients with risk factors for invasive candidiasis and no other known cause of fever. Fluconazole or an echinocandin are the drugs of choice. ${ }^{33}$ An echinocandin or liposomal amphotericin B is preferred in most of neutropenic patients with recent exposure to fluconazole or at high risk of infection due to Candida glabrata or Candida krusei, resistant to azole. Moreover fluconazole shows less activity against $C$. glabrata or $C$. krusei than against other Candida spp. Voriconazole can be used in situations in which additional coverage for mold is desired. ${ }^{33}$

Guidelines provided by the third European Conference of Infections in Leukemia (ECIL 3) recommended the use of liposomal amphotericin B, caspofungin or voriconazole in neutropenic patients before species identification, whereas fluconazole should not be used due to prior exposure to fluconazole as prophylaxis. Amphotericin B deoxycholate is generally not recommended in hematological patients as the concomitant use of nephrotoxic drugs or renal impairment is more frequent. After species identification, caspofungin and liposomal amphotericin B are considered the primary choice for $C$. glabrata and $C$. krusei while voriconazole may be considered an alternative treatment. ${ }^{34}$ ECIL 3 also recommends to continue antifungal therapy for candidemia in neutropenic host at least for 14 days after the last positive blood culture, resolution of signs and 
symptoms and resolved neutropenia. ${ }^{34}$ The strong recommendation to remove central venous line in non-hematological patients, loses out in neutropenic patients in which catheter removal often creates substantial intravenous access difficulties. However experts' opinion is that catheter should be removed when it is possible. On the other hand, removal of central venous line is always recommended when $C$. parapsilosis is isolated. 34

In conclusion, the medical treatment of candidemia is changed in the last 2 decades with the introduction of safe and broad-spectrum antifungal drugs. This allows to adjust the antifungal therapy on the basis of patient clinical conditions and comorbidity, previous history of antifungal prohylaxis, and empirical or target use of antifungals. More studies are needed in the pediatric setting to tailor the therapy not only on epidemiology and risk factors, but also on pharmacokinetic properties of new molecules.

\section{References}

1. Zaoutis TE, Argon J, Chu J, et al. The epidemiology and attributable outcomes of candidemia in adults and children hospitalized in the United States: a propensity analysis. Clin Infect Dis 2005;41:1232-9.

2. Martin GS, Mannino DM, Eaton S, Moss M. The epidemiology of sepsis in the United States from 1979 through 2000. N Engl J Med 2003;348:1546-54.

3. Wisplinghoff $\mathrm{H}$, Seifert $\mathrm{H}$, Tallent SM, et al. Nosocomial bloodstream infections in pediatric patients in United States hospitals: epidemiology, clinical features and susceptibilities. Pediatr Infect Dis J 2003;22: 686-916.

4. Kao AS, Brandt ME, Pruitt WR, et al. The epidemiology of candidemia in two United States cities: results of a population based active surveillance. Clin Infect Dis 1999;29: 1164-70.

5. Hajjeh RA, Sofair AN, Harrison LH, et al. Incidence of bloodstream infections due to Candida species and in vitro susceptibilities of isolates collected from 1998 to 2000 in a population based active surveillance program. J Clin Microbiol 2004;42:1519-27.

6. Manno G, Scaramuccia A, Rossi R, et al. Trends in antifungal use and species distribution among Candida isolates in a large paediatric hospital. Int J Antimicrob Agents 2004;24:627-8.

7. Watson RS, Carcillo JA, Linde-Zwirble WT, et al. The epidemiology of severe sepsis in children in the United States. Am J Respir Crit Care Med 2003;167:695-701.

8. Kaya Z, Gursel T, Kocak U, et al. Invasive fungal infections in pediatric leukemia

Table 1. Pediatric dosages and grade of recommendation of different antifungals for neutropenic patients

\begin{tabular}{|c|c|c|c|c|c|}
\hline & $\begin{array}{l}\text { Licensed } \\
\text { for pediatric } \\
\text { patients }\end{array}$ & $\begin{array}{l}\text { Pediatric } \\
\text { dosages }\end{array}$ & $\begin{array}{l}\text { IDSA } \\
\text { score }\end{array}$ & $\begin{array}{l}\text { IDSA score } \\
\text { in neonates }\end{array}$ & $\begin{array}{l}\text { ECIL } 3 \\
\text { score }\end{array}$ \\
\hline Fluconazole* & Yes & $8-12 \mathrm{mg} / \mathrm{kg} / \mathrm{d}$ qd & BIII & BII & CIII \\
\hline $\begin{array}{l}\text { LFAmB (lipid } \\
\text { amphotericin B } \\
\text { formulation) }\end{array}$ & Yes & $3-5 \mathrm{mg} / \mathrm{kg} / \mathrm{d}$ qd & AII & $\mathrm{BII}^{\circ}$ & BII \\
\hline $\begin{array}{l}\text { AmB-d } \\
\text { (amphotericin B } \\
\text { deoxycholate) }\end{array}$ & Yes & $0,5-1 \mathrm{mg} / \mathrm{kg} / \mathrm{d}$ qd & - & AII & CIII \\
\hline Caspofungin & Yes & $\begin{array}{l}\text { Day } 170 \mathrm{mg} / \mathrm{m}^{2} \\
\text { then } 50 \mathrm{mg} / \mathrm{m}^{2} / \mathrm{d} \text { qd }\end{array}$ & AII & BIII $^{\#}$ & BII \\
\hline Micafungin & Yes & $2-4 \mathrm{mg} / \mathrm{kg} / \mathrm{d}$ qd & AII & $\mathrm{BIII}^{\#}$ & BII \\
\hline Anidulafungin & No & $1,5 \mathrm{mg} / \mathrm{kg} / \mathrm{d}-$ & AIII & - & BII \\
\hline Voriconazole & Yes & $\begin{array}{c}14 \mathrm{mg} / \mathrm{kg} / \mathrm{d} \text { bid } \\
>12 \text { yrs } 200 \mathrm{mg} \\
\text { bid+TDM§ }\end{array}$ & $\mathrm{BIII}^{\wedge}$ & - & BII\$ \\
\hline
\end{tabular}

IDSA, Infectious Diseases Society of America; ECIL 3, third European Conference of Infections in Leukemia. ${ }^{*}$ Not in severely ill patients or in patients with previous azole exposure. 'If urinary tract involvement is excluded. \#In case of resistance to fluconazole or AmB-d toxicity. \&If additional mold coverage is desired. ${ }^{\wedge}$ Therapeutic drug monitoring. ${ }^{\$}$ Not in patients with previous azole exposure

patients receiving fluconazole prophylaxis. Pediatr Blood Cancer 2009;52:470-5.

9. Rosen GP, Nielsen K, Glenn S, et al. Invasive fungal infections in pediatric oncology patients: 11-year experience at a single institution. J Pediatr Hematol Oncol 2005;27:135-40.

10. Castagnola E, Cesaro S, Giacchino M, et al. Fungal infections in children with cancer: a prospective, multicenter surveillance study. Pediatr Infect Dis J 2006;25:634-9.

11. Mor M, Gilad G, Kornreich L, et al. Invasive fungal infections in pediatric oncology. Pediatr Blood Cancer 2011;56:1092-7.

12. MacDonald L, Baker C, Chenoweth C. Risk factors for candidemia in a children's hospital. Clin Infect Dis 1998;26:642-5.

13. Dvorak CC, Steinbach WJ, Brown JM, Agarwal R. Risks and outcomes of invasive fungal infections in pediatric patients undergoing allogeneic hematopoietic cell transplantation. Bone Marrow Transplant 2005;36:621-9.

14. Zaoutis TE, Prasad PA, Localio AR, et al. Risk factors and predictors for candidemia in pediatric intensive care unit patients: implications for prevention. Clin Infect Dis 2010;51:e38-45.

15. Blyth CC, Chen SC, Slavin MA, et al. Not just little adults: candidemia epidemiology, molecular characterization, and antifungal susceptibility in neonatal and pediatric patients. Pediatrics 2009;123:1360-8.

16. Horn DL, Neofytos D, Anaissie EJ, et al. Epidemiology and outcomes of candidemia in 2019 patients: data from the prospective antifungal therapy alliance registry. Clin Infect Dis 2009;48:1695-703.

17. Pappas PG, Rex JH, Lee J, et al. A prospective observational study of candidemia: epidemiology, therapy, and influences on mortality in hospitalized adult and pediatric patients. Clin Infect Dis 2003;37:63443.

18. Mikulska M, Calandra T, Sanguinetti M, et al. Third European Conference on Infections in Leukemia Group. The use of mannan antigen and anti-mannan antibodies in the diagnosis of invasive candidiasis: recommendations from the Third European Conference on Infections in Leukemia. Crit Care 2010;14:R222.

19. Garey KW, Rege M, Pai MP, et al. Time to initiation of fluconazole therapy impacts mortality in patients with candidemia: a multi-institutional study. Clin Infect Dis 2006; 43:25-31.

20. Kullberg BJ, Sobel JD, Ruhnke M, et al. Voriconazole versus a regimen of amphotericin B followed by fluconazole for candidaemia in non-neutropenic patients: a randomised non-inferiority trial. Lancet 2005;366:1435-42.

21. Rex JH, Bennett JE, Sugar AM, et al. A randomized trial comparing fluconazole with amphotericin B for the treatment of candidemia in patients without neutropenia. Candidemia Study Group and the National Institute. N Engl J Med 1994;331:1325-30.

22. Mora-Duarte J, Betts R, Rotstein C, et al. Comparison of caspofungin and amphotericin B for invasive candidiasis. N Engl J Med 2002;347:2020-9.

23. VandenBussche HL, Van Loo DA. A clinical review of echinocandins in pediatric patients. Ann Pharmacother 2010;44:16677.

24. Ikeda F, Saika T, Sato Y, et al. Antifungal activity of micafungin against Candida and Aspergillus spp. isolated from pediatric 
patients in Japan. Med Mycol 2009;47:1458.

25. Natarajan G, Lulic-Botica M, Rongkavilit C, et al. Experience with caspofungin in the treatment of persistent fungemia in neonates. J Perinatol 2005;25:770-7.

26. Merlin E, Galambrun C, Ribaud P, et al. Efficacy and safety of caspofungin therapy in children with invasive fungal infections. Pediatr Infect Dis J 2006;25:1186-8.

27. Groll AH, Attarbasc A, Schuster FR, et al. Treatment with caspofungin in immunocompromised paediatric patients: a multicentre survey. J Antimicrob Chemother 2006;57:527-35.

28. Kuse ER, Chetchotisakd P, da Cunha CA, et al. Micafungin versus liposomal amphotericin B for candidaemia and invasive candidosis: a phase III randomised doubleblind trial. Lancet 2007;369:1519-27.

29. Pappas PG, Rotstein CMF, Betts RF, et al. Micafungin versus caspofungin for the treatment of candidemia and other forms of invasive candidiasis. Clin Infect Dis 2007;45:883-93.

30. Queiroz-Telles F, Berezin E, Leverger G, et al. Micafungin versus liposomal amphotericin B for pediatric patients with invasive candidiasis: substudy of a randomized double-blind trial. Pediatr Infect Dis J 2008;27:820-6.

31. Ostrosky-Zeichner L, Kontoyiannis D, Raffalli J, et al. International, open-label, noncomparative, clinical trial of micafungin alone and in combination for treatment of newly diagnosed and refractory can- didemia. Eur J Clin Microbiol Infect Dis 2005;24:654-61.

32. Reboli AC, Rotstein C, Pappas PG, et al. Anidulafungin versus fluconazole for invasive candidiasis. N Engl J Med 2007;356: 2472-82.

33. Pappas PG, Kauffman CA, Andes D, et al. Clinical practice guidelines for the management of candidiasis: 2009 update by the Infectious Diseases Society of America. Clin Infect Dis 2009;48:503-35.

34. Maertens J, Marchetti 0, Herbrecht R, et al. European guidelines for antifungal management in leukemia and hematopoietic stem cell transplant recipients: summary of the ECIL 3--2009 update. Bone Marrow Transplant 2011;46:709-18. 